\title{
Implementing Open Science Policies into Library Processes - Case Study of the University of Eastern Finland Library
}

\section{Jarmo Saarti}

University of Eastern Finland Library

jarmo.saarti@uef.fi, orcid.org/0000-0001-6565-0969

\section{Tomi Rosti}

University of Eastern Finland Library

tomi.rosti@uef.fi, orcid.org/0000-0003-2098-8389

\section{Helena Silvennoinen-Kuikka}

\author{
University of Eastern Finland Library \\ helena.silvennoinen-kuikka@uef.fi
}

\begin{abstract}
This is a case study about the creation of open science services in the University of Eastern Finland. The library has overseen the open science services that have been actively implemented from 2010 onwards due to the development of the digitalisation of science and open science policies. A survey was conducted to determine how the UEF's academic faculty use the services provided as well as their attitudes towards opening their own research findings in this manner. The researchers seem to be most interested in issues that influence their daily work, i.e. data management plans and opening their publications. It seems that the culture of openness is still at the development stage within UEF. The innovators, i.e. active research groups and researchers, are already practicing and encouraging openness, but the
\end{abstract}


majority of the academic staff seems to be either unaware of open science or unwilling to implement it, due to the fact that incentives and career advancements still support the traditional way of conducting research.

Keywords: open science; open access; open science services; academics; academic libraries

\section{Introduction}

The promotion of open access to research publications has been rapidly progressing within the European Union and its Member States. Public research funding agencies have increasingly called for the adoption of transparency in the research they fund. (European Commission, 2017.) In fact, transparency is being promoted in the same way, both politically and operationally, throughout the world. Transparency has traditionally referred to transparency in funding and in the ethical use of the allocated resources. Nonetheless, a full opening of data, publications and even methods seems to increase not only transparency but also the impact of the actual research conducted with publicly funded projects.

Open Access business models are still being developed, but there is already evidence that open publishing is becoming the mainstream of scientific publishing (Archambault et al., 2014). In the last couple of years, a requirement for transparency has also been imposed on research data.

Today, openness and transparency are at the forefront of research development and in recent years, for example, data mining and machine learning have yielded significant research results in many disciplines. In fact, it seems that these new technologies are already capable of transcending the ability of humans to process data (Clifton, 2019; a good example of the technology's new possibilities is ScienceDirect's auto-generated web-page about data mining research published in ScienceDirect (2020)). These kinds of developments pose new challenges for the staff and management of scientific libraries. At the same time, the demand for new types of services has increased.

Ideally, science should aim for full openness: first, this allows science to progress since published findings are subjected to critical review, and secondly, 
it is the only way in which all of mankind can reap the benefits of science. (Saarti \& Tuominen, 2017.) Nonetheless, openness must be carried out in an ethical and sustainable manner and it should not affect negatively on an academic's career prospects or on an individual's protection of privacy. It is self-evident that the development of research findings can be accelerated by making both research publications and the research data as widely accessible as possible. Scientific data is also extensively used by both public and commercial actors. A good example of this is spatial information and its use in different contexts.

However, building the ideal situation still requires the implementation of several aspects, e.g., developing the services provided by libraries and changing the business models operated by scientific publishers. In addition, scientific communities must develop new tools, technologies, and methods to allow them to function in this new operational environment. In terms of learning, not only teaching new skills to students but also retraining the entire academic community will be needed.

The movement of promoting open science began in the 1990s. It may be argued, however, that at first it was more an ideological rhetoric rather than offering practical solutions for academic work (Ilva, Laitinen, \& Saarti, 2016). The scholarly publishing of articles behind paywalls changed the operational environment of academic libraries. At the same time, article copyright license agreements began to determine who could read scientific articles - were they openly available online or behind a paywall.

From the beginning of the current millennium, politicians and other decisionmakers began to contribute to the debate and favour open science. Initially, the aim was to provide open access to publications funded by public research funding. Activities changed dramatically when funding agencies began to require open access as a prerequisite for research funding - although initially this was expressed only as a recommendation. (See e.g. European Commission, 2012).

A new way of distributing research publications emerged: libraries and similar actors began to set up repositories for self-archiving storage. Selfarchiving refers to the process by which a publication that is placed behind a paywall by a commercial publisher in addition is stored for public use on an open access information system, usually in the form of a manuscript. 
Recently, discussions on open science have largely concerned the opening of research data and related analytical methods and software with the goal of enabling data quality assessment and reuse (EOSC Summit, 2017; European Commission, 2018, 33; Kraker, Leony, Reinhardt, \& Beham, 2011). It seems that open data and its management will become a part of academic library collections and activities, or at least libraries will facilitate the opening process in one way or another. The data sets are already very extensive; the challenges of managing them, especially concerning their long-term storage, will be huge (see e.g., European Commission, 2017). Moreover, it can already be seen that opening and retaining different types of open science resources will require significant additional resources. The concept of cost-free open science is a complete nonsense; cost management also requires common resources, a challenge that the European Open Science Cloud (EOSC) initiative has started to address in the EU with the aim of providing a common service platform for access to European research results and data (European Commission, 2019).

The Association of European Research Libraries (LIBER, 2018) and the League of European Research Universities (LERU, 2018) have published open science roadmaps. There are several recommendations to help organisations make their research activities more open, e.g. to collaborate and build services based on a sustainable business model. Coordinating the process is essential as multiple actors will be needed to make the change a reality. For example, changing the merit and reward system requires a common vision throughout the global research community, not only on the European, national, or even the local level.

In 2017, the co-operation organisation for the Finnish Universities (UNIFI) created a workgroup to find solutions for the management of open science issues. The workgroup published a report in summer 2018 with a recommendation to establish a national level coordination body. The coordination was entrusted to the Federation of Finnish Learned Societies. By the end of 2018, four expert sub-groups had been established to support the open science progress: open data, open publishing, open learning, and open research community.

Table 1 shows the main clusters of open science that require attention and services from the UEF library. It can already be seen that the opening of research publications has become a routine part of the library's work. This 
Table 1: The multifaceted operational environment of open science for the UEF library.

\begin{tabular}{lll}
\hline Open publications & Open data & Open science and open learning \\
\hline - Open access & - Opening data & - Born digital research data and resources \\
publishing & - Methods and & - Digitized research data and resources \\
- Self-archiving & source codes & - Learning resources, learning software, \\
- Simultaneous open & - Metadata and & open data from learning, virtual open \\
and closed publishing & linked data & learning environments \\
\hline
\end{tabular}

Metrics and methods

is a relatively easy task to manage for libraries since the rules and practices of research publishing are familiar and well-regulated. The management of opened data and learning resources is already a growing part of the library's tasks, although still a minor one. All these demand new types of metrics and methods that are being developed as the services emerge. Other areas of open science and the role of different actors are still developing. The current stage of development, with its various services and practices, seems somewhat chaotic, although some evidence has been presented that common and uniform services are being created both at the international and national levels (Ogungbeni, Obiamalu, Ssemambo, \& Bazibu, 2016; Saarti, 2018).

It also seems that, due to the rapid evolution of the digital environment, the future changes will be long-lasting and even paradigmatic in their manner. It gives libraries an opportunity to influence and introduce their long-term best practices and experiences to a new environment. Scientific publications must be accessible for a long period of time and they must be easy to access. In addition, up-to-date technologies and methods should be utilised for data mining; these are techniques with which libraries are experienced.

\section{The Building of Open Science Services at the UEF Library}

In 2014, the Ministry of Education and Culture of Finland released the Open Science and Research Roadmap for the years 2014-2017. The goal for Finland was to become a leading country for openness in science and research by 2017. At the same time, the Ministry launched a million-euro funding call for new ideas and approaches for promoting open science. 
The University of Eastern Finland (UEF) and the University of Jyväskylä were granted funding by the Ministry for a project: SURIMA 2015-2017, Finland a model country for green open access. The purpose of the project was to build a model process for self-archiving scientific publications within Finnish academic institutions. As an outcome of the project, a repository for green open access publications was launched in the University of Eastern Finland in 2016. Since 2018, the same repository has been used as a metadata repository for research data.

The SURIMA project, along with the more comprehensive Open Science and Research Roadmap 2014-2017 by the Ministry of Education and Culture of Finland, raised general awareness among the library staff of the broad range of issues concerning open science. By 2015, the need for developing research support and open science services at the UEF library became evident.

In 2016, a one-year project on research support and open science services was launched in the UEF library. Almost half of the total staff, in addition to their regular duties, were involved in this project. The main purpose of the project was to enhance the library's role in promoting open science and in supporting the university's researchers. A project coordinator was nominated, and 5 thematic teams were devised:

- Open access publishing

- Open research data

- Research assessment

- Library resources and information literacy

- Research visibility

The project was initiated by increasing the knowledge of the project members about open science and research support issues. The UEF library was benchmarked against other university libraries, both in Finland and in other countries. Team members attended national conferences and seminars, reviewed open science policy papers and strategies in addition to numerous national and international websites to obtain a better understanding of the various aspects of open science.

It soon became evident that open science would require an expansion of traditional library services and the adoption of new roles. The development of new open science and research support services, infrastructures and tools 
would also require qualifications beyond those of traditional library skills. Since 2016, four new employees have been recruited, with three of them having a PhD degree and a good knowledge of research and educational processes. A library degree was no longer deemed necessary for these new positions as had been the case for the recruitments made in previous years. In addition to these new recruits, many existing library professionals have adopted new responsibilities and roles to promote open science.

Open science is transforming the work done by the library. The development of open scientific tools and the creation of research support services need to be based on the personnel's implementation skills and abilities to work in institutional, national, and international multi-professional teams and networks. It is worth mentioning that all the library staff should have a basic knowledge of the developments taking place in open science. This is important not only for keeping up their professional skills, but also for the sense of community and workplace spirit. In 2018, all library workers in the UEF attended an eight-session in-house training organised by the open science and research support specialists in the library.

At the University level, the work towards full openness started with the open access publishing. This has meant the start of a self-archiving repository (https://erepo.uef.fi/), allocations to open access publishing fees including a requirement listed in the national level consortium agreements for the possibility to publish openly. The library collects the metadata of the opened datasets into its repository. The work towards open learning resources has started at the national level with the implementation of a national portal for open access learning resources (https://aoe.fi/). The UEF has created its open infrastructure work with pilot projects and is working at the present on an infrastructure strategy. Work in all these areas has also involved extensive instruction and guidance for both the academic staff and students.

\subsection{Co-creating and Implementing University Services and Policies}

In 2017, the library was nominated by the Rector of the University of Eastern Finland to co-ordinate the University's open science services. This seemed natural as since the beginning of 2010, the library had been active in promoting open publishing and data policy creation together with the University 
administration. The publishing of UEF publishing and data policy in 2015 saw the launch of the open science service which was a collaboration between the experts in the library, IT services and the UEF's administration.

Collaborative actions have included roadshows on open science themes for the different faculties as well as the creation of a new strategy and an open science action plan for the University of Eastern Finland. The service building process has been reinforced by reallocating current work resources and by recruiting new staff supported through the yearly budget negotiations and project funding.

\subsection{Library's Role and Tasks in Providing Services for Open Science}

At the present, i.e. in 2020, open science and research support issues are embedded in the annual action plan and the activities of the UEF library. The library is committed to supporting researchers to undertake research by adopting an open approach. However, the concept of research support services is often preferred instead of the concept of open science services. The reason for this situation is that there still is, and most probably always will be, some research data that cannot be opened due to legislative restrictions, e.g. privacy protection laws. It has also been noted that many researchers are still not familiar with the concept of open science, but they all understand the concept of research support. This article focuses on the services where open science issues are present.

Research support services in the UEF library cover practices in publishing, research data, and research metrics. The library has also adopted the role of an active advocate and a trainer of open science issues within the UEF University.

The publication services cover:

- UEF-eRepository services for self-archiving green open access publications,

- registration of the publications of the University of Eastern Finland in the SoleCris research database,

- UEF Electronic Publication service for open access publishing at the University of Eastern Finland, 
- supporting and counseling researchers in open access publishing while using Article Processing Charges (APC). Since the beginning of 2019, the UEF library has been monitoring the APC expenses in the University of Eastern Finland.

Research data services cover:

- UEF-eRepository service for the metadata of research data made available by the UEF researchers in various data archives,

- Data Management Plan (DMP)-tool,

- supporting and counseling researchers in the management of research data (e.g. data archiving, metadata, DMPs, Copyright licenses, identifiers),

- DMP-workshops for researchers.

Research metrics services cover:

- bibliometric analyses on request,

- supporting and counseling researchers and the university administration in research metrics.

The UEF library supports open science also by being an open science advocate and trainer of students and researchers. The library practices cover:

- communication services about open science via a variety of channels within the University of Eastern Finland (webpages, social media, working committees etc.),

- co-development of open science strategies, infrastructures, and guidelines at national and institutional working committees,

- embedding open science elements in the contents of library courses for undergraduate students and $\mathrm{PhD}$ - students,

- training and counseling researchers and communicating about library's research support and open science services.

Owing to the library's active role in advocating for open science, the library is often seen as a "jack of all trades" or a general helpdesk service of open science issues in the university. However, the library per se does not have resources, authority nor competence to cover all the aspects of open science in research and education processes. Instead, the library recommends that the processes should be re-examined and re-defined at the university level. After clarifying the research process, then the roles, practices, 
and resources could be determined between the various actors within the university.

The UEF library has exerted significant efforts and resources on providing research support and open science services during the past 5 years. However, there are still many challenges ahead. Many researchers are not yet familiar with open science, others are also unwilling to publish in open access publications if the credit system of researchers is dependent on publishing in traditional, i.e. commercial, scientific publications.

\section{Survey and its Analysis}

\subsection{Background \& Research Questions}

The purpose of the survey was to collect data from the academics in the University of Eastern Finland to evaluate the following:

1. what is the know-how about the open science,

2. what support services are currently being used, and

3. what motivates the opening of research within the UEF?

We constructed a simple survey questionnaire that consisted of 10 multiple choice questions and one open question for general feedback. Questions 1 to 3 were background questions about age, faculty, and career status. Questions 4 to 10 were as follows:

- Have you attended training or workshops on any of the open science issues organised by the University of Eastern Finland after the year $2017 ?$

- Have you attended training or workshops organised by any of the UEF units?

- Have you opened your research process and if so, how?

- What is your motivation to move to open science?

- How extensive is the opening of the research process in your working environment?

- Where have you received help in open science over the past year?

- Have you received advice or guidance in promoting open science? 
The questionnaire was sent via e-mail to all of the academics at the UEF $(\mathrm{N}=1,735)$. We received a total of 261 responses $(15.0 \%)$. The answers were analysed by descriptive statistics and are presented in the following chapter. Due to the nature of the survey, one must be careful in interpreting the results, especially when trying to generalise these findings to situations outside of UEF.

The age groups of the respondents were divided rather evenly in the various decades. The oldest and youngest groups each accounted for about $20 \%$ of the respondents, the largest age groups had been born in the years 1970-1979 and 1980-1989, both about 30 per cent (see Figure 1) of the entire number of the respondents.

The respondents' number per faculty was also quite evenly divided (see Figure 2). The four faculties of the UEF are about the same size. We obtained most answers from the Faculty of Health Sciences and from the Faculty of Science and Forestry - both about 30 per cent. The Faculty of Social Sciences and Business and the Philosophical Faculty were represented by about $20 \%$. One may speculate that this also illustrates the faculties' maturity level with respect to open science. For example, the Faculty of Science and Forestry, e.g. physics, has a longer tradition in opening its scientific work.

Fig. 1: Respondents by age groups ( $N=261$ persons).

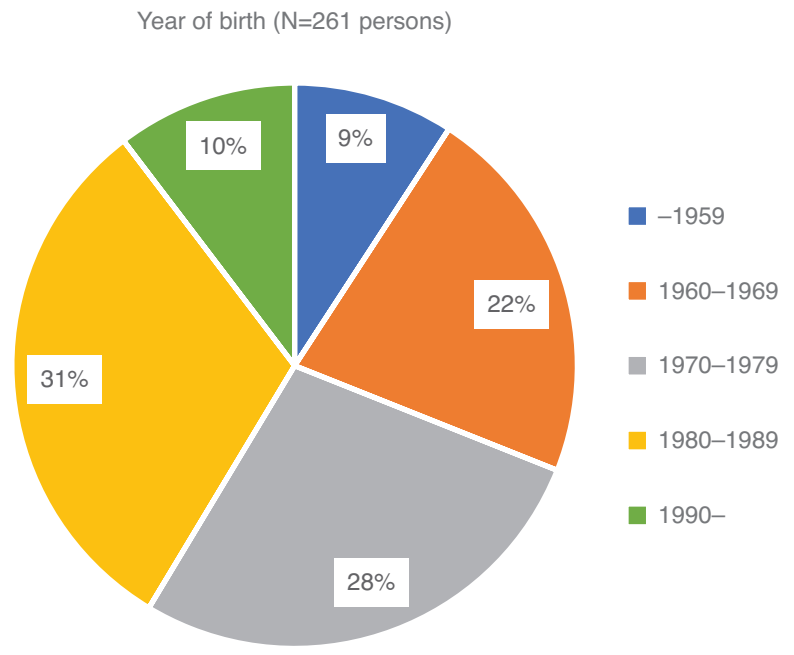


Fig. 2: Respondents by faculties ( $N=261$ persons).

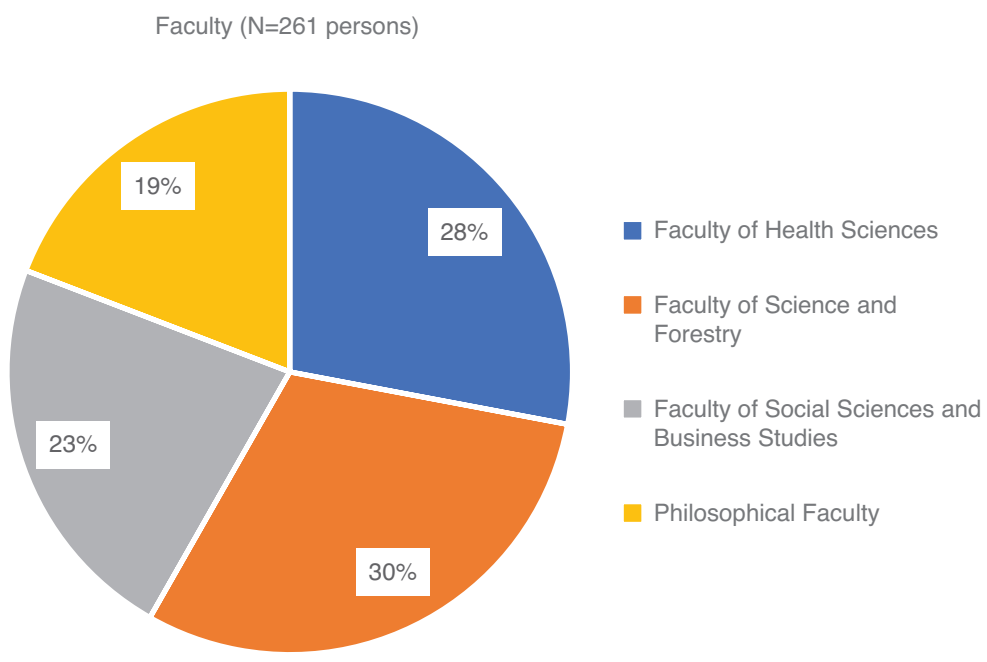

The division by career stages was also rather evenly distributed. The most active level, e.g. researchers and senior researchers, accounts for about 30 per cent of the respondents. This group is often also responsible and active in managing the university's research groups. Doctoral students and assistants comprised the second largest level with a share of 26 per cent. The professors and postdoctoral students each represented about 20 per cent of the total number of respondents (see Figure 3).

\subsection{Results}

The first question on open science dealt with attendance at the training or workshops at any of the open science issues organised by the University of Eastern Finland after 2017. As can be seen in Figure 4, most of the respondents - about $80 \%$ - had not attended any of the training sessions provided by the university. Among the attendants, the best attended courses dealt with open access publishing and opening one's own research data, about $10 \%$ of attendance rate for each.

When asked about the provider of the training, the faculties and the library seem to be the most extensively accessed providers, followed by research services and IT services. However, the clear majority of the respondents had not attended any training or workshops at all. (see Figure 5). 
Fig. 3: Respondents by career stage ( $N=261$ persons).

Career stage ( $\mathrm{N}=261$ persons)

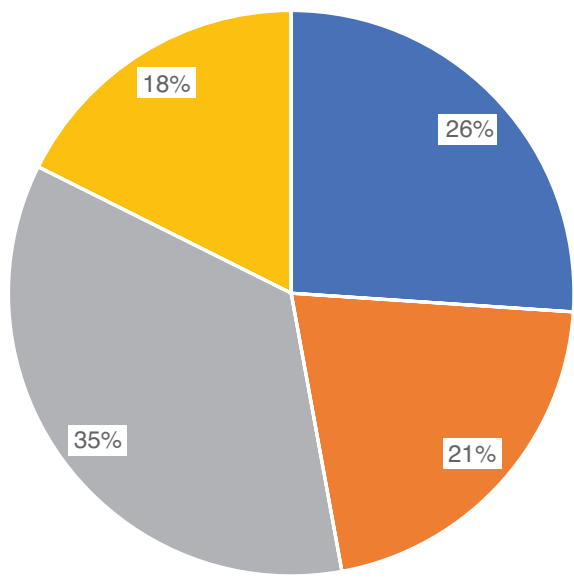

Level 1 - e.g. doctoral student, specialising doctor/dentist, research assistant, teaching assistant

Level 2 - e.g. postdoctoral researcher, university instructor

Level 3 - e.g. university lecturer and clinical instructor, university researcher, senior researcher, research coordinator and curator, assistant professor (tenure track)

Level 4 - e.g. professor, research director and senior curator

Fig. 4: Respondents' attendance in the training sessions ( $N=261$ persons).

Have you attended training or workshops on any of the open science issues organised by the University of Eastern Finland after 2017? ( $\mathrm{N}=261$ persons)

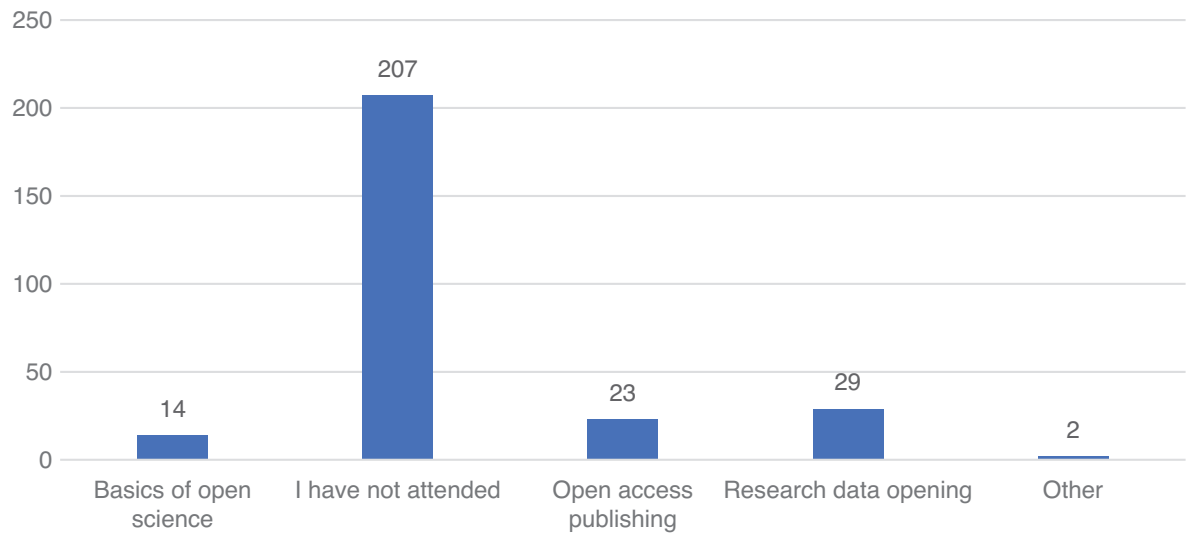

When asked in what way the respondents had opened their research work, open access publishing was, as expected, the principal way of opening one's research: $66.7 \%$ of the respondents had opened their publications. On the other hand, $26.1 \%$ of the respondents had not opened their research in any way. 
Fig. 5: Training providers ( $N=261$ persons).

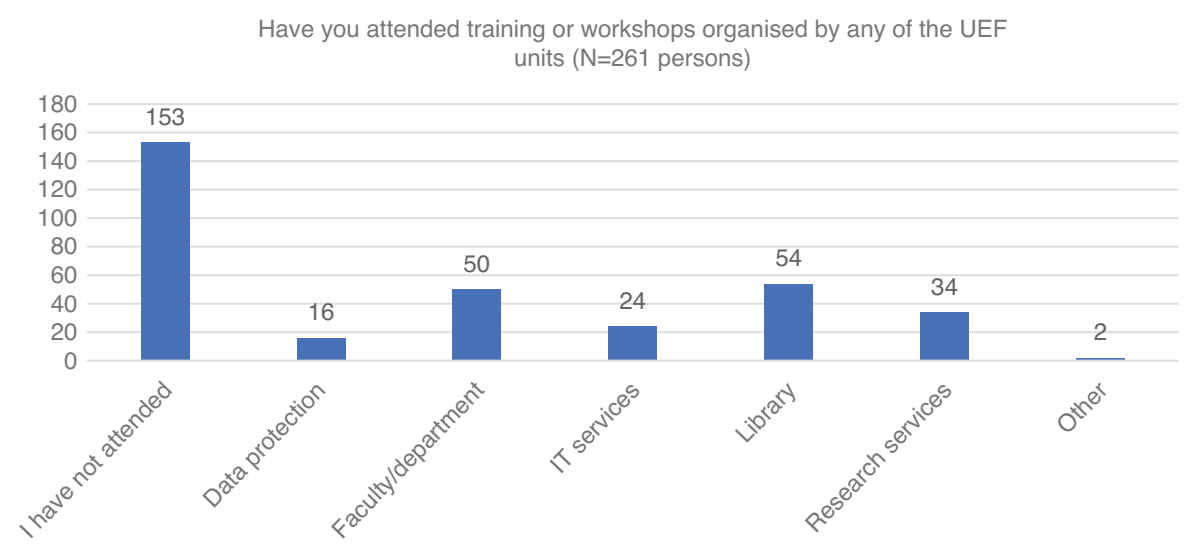

Opening research data, methods and teaching materials are still less extensively used ways of opening one's research work, only about $20 \%$ each (see Figure 6).

When asked about the motivation for opening one's research work, the inner need was mentioned as the most important factor (52.5\%) (see Figure 7).

Fig. 6: What resources have the respondents opened ( $N=261$ persons).

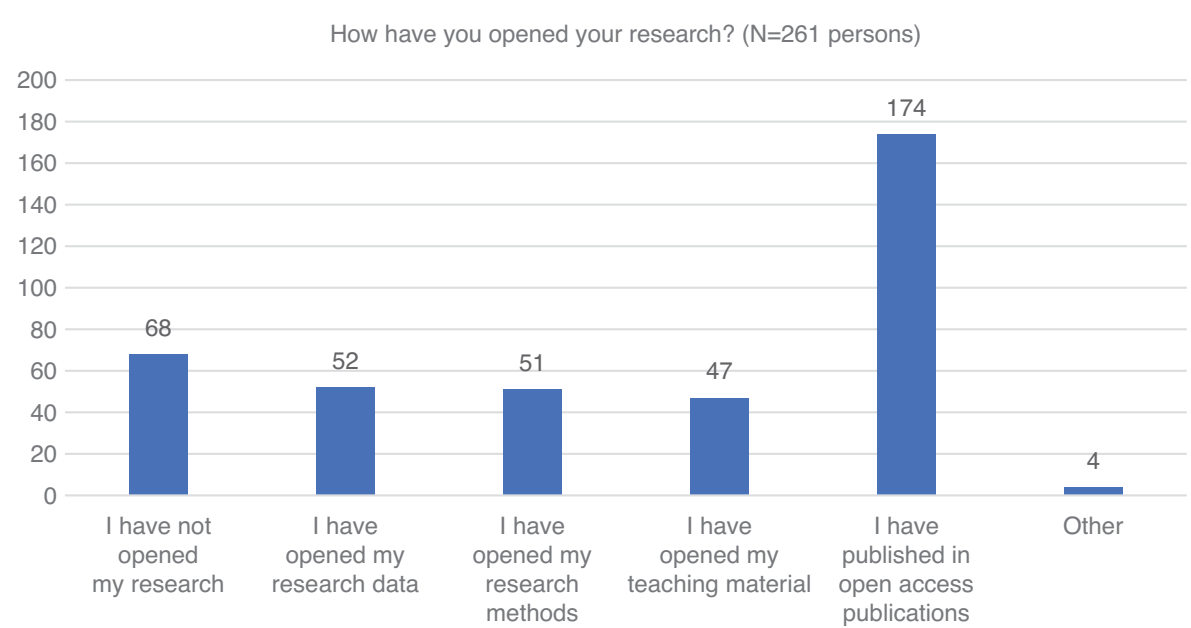


Fig. 7: What motivates the respondents ( $N=261$ persons).

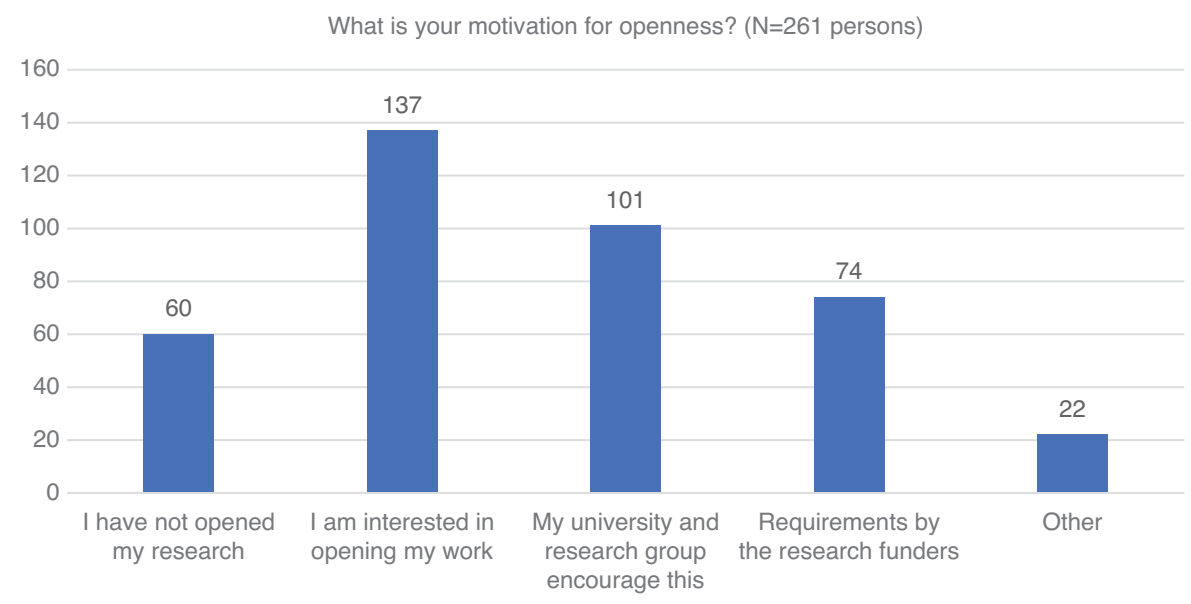

Almost equally important was the research group's motivation and requirements set by the funders.

The open answers supported the notion that it was the researcher's own motivation which was very important, and this form of openness was viewed as a normal way of conducting research: "It is the only way to ensure the reproducible research .... It is ethical. ... I am interested in opening my work ... I see it as a fundamental principle of scientific research."

When asked about how extensive the opening of the research was in one's working environment, the most important motivational reference group appeared to be the research group (42.1\%) (see Figure 8). The second, and almost equally important, was department level motivation. It seems that the faculty level strategic work is either not viewed as so important or it is not affecting the level where the research is being performed in the university.

When asked about where the respondents had received help in open science issues over the past year, the clear majority answered that they had not asked for any help (Figure 9). Those in need had used mostly the library's services $(25.3 \%)$. In addition, the department level guidance was of some importance. Other services were not used frequently, or they played a minor role. 
Fig. 8: The extensiveness of the opening of the research ( $N=261$ persons).

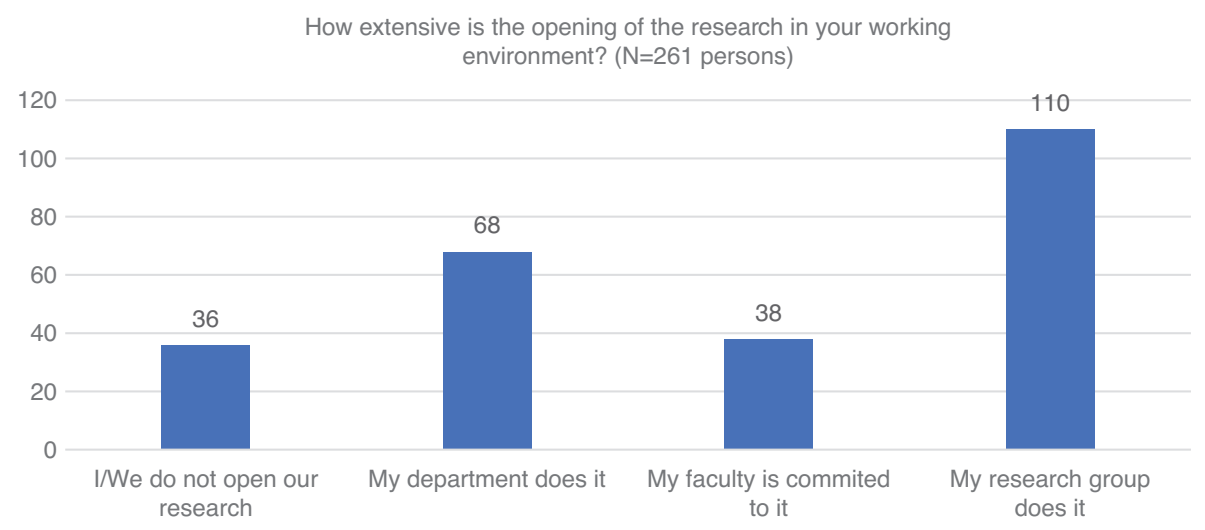

Fig. 9: The open science help providers ( $N=261$ persons).

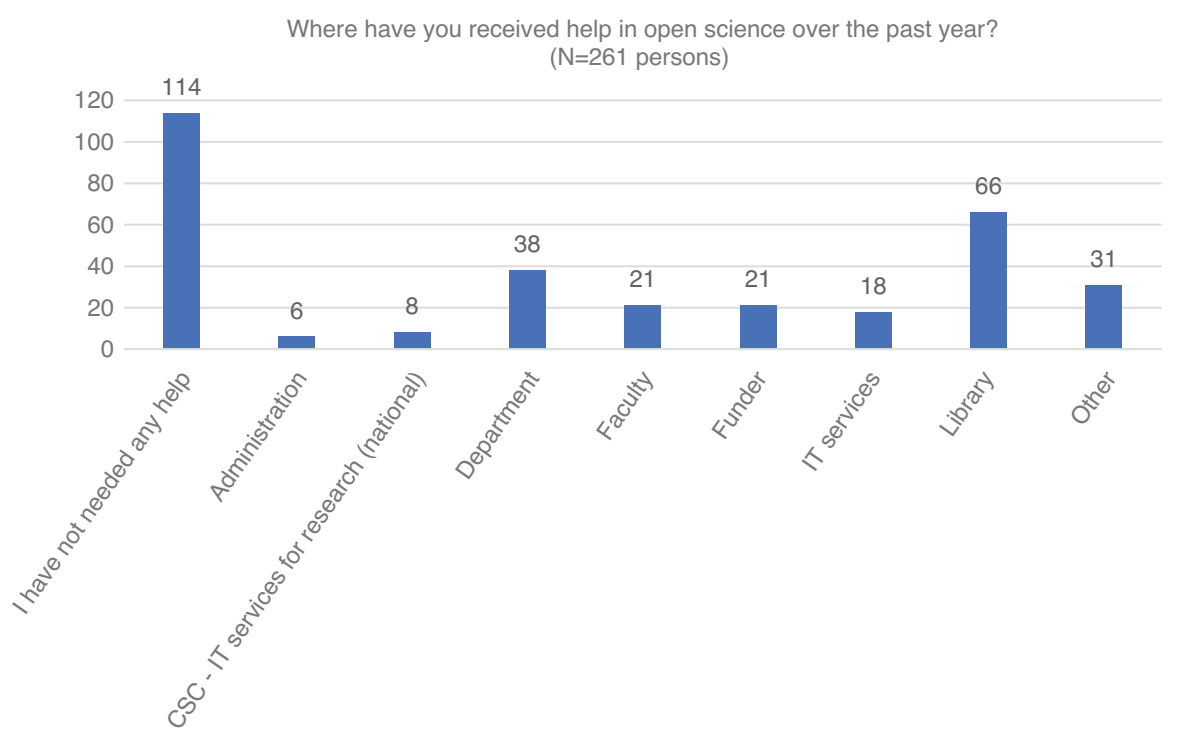

The greatest need for guidance concerned data management plans and open access publishing, most likely due to the requirements of the principal Finnish and global funders. Another major issue where the respondents had needed help was the visibility of the researcher, e.g. how does the individual researcher receive credit in these forums (see Figure 10). 
Fig. 10: Help services by themes ( $N=261$ persons).

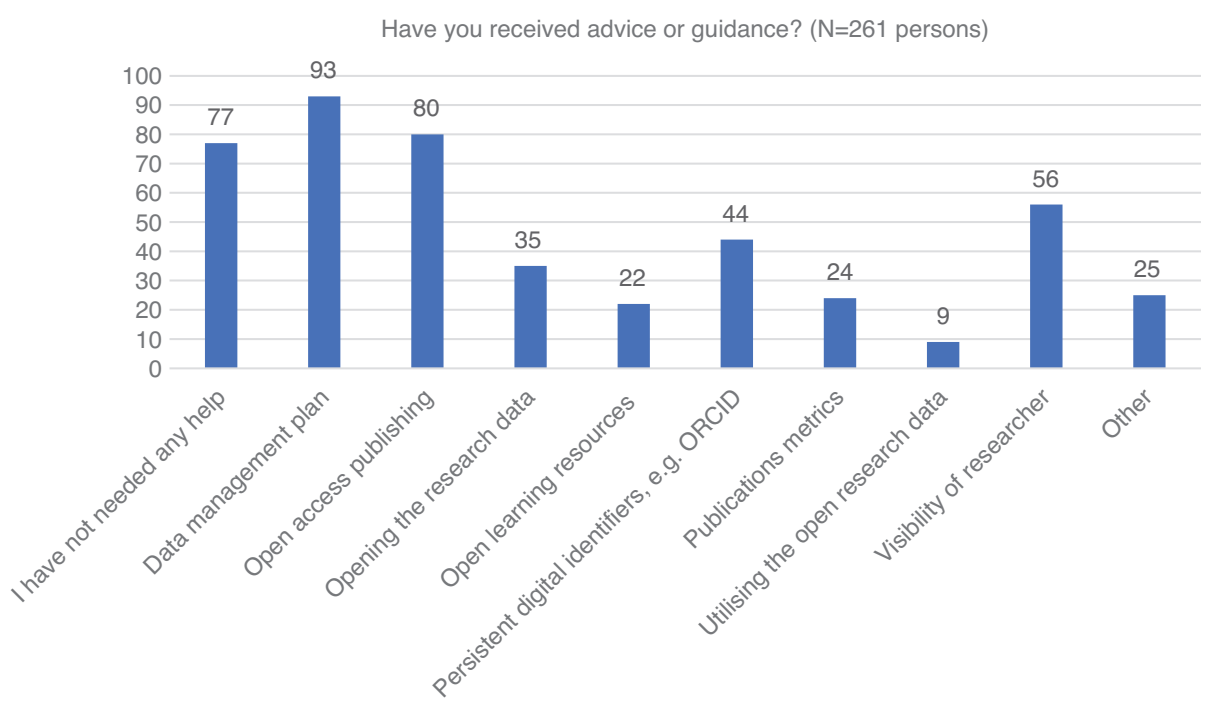

Based on the answers collected from the respondents, the actual opening of data and the reuse of open data is not yet a part of the research activities being conducted within the UEF. This can also be seen from the UEF open data repository where the number of open datasets is relatively modest, at the time being only 159 files (see: https:/ / erepo.uef.fi/handle/123456789/6334/ browse?type=title).

The open answers strengthen the concept that there is a knowledge gap among the researchers: "is there 'advice and guidance' for this? ... I have not received help! ... not yet, but I would like to receive guidance in the above issues ... Wish to receive guidance about the above-mentioned topics".

\section{Conclusions}

Based on the recent report on the evaluation of openness in the activities of higher education institutions (Forsström, Lilja, \& Ala-Mantila, 2020) and on the results of the present survey, it seems that the University of Eastern Finland and its library have succeeded in the strategical work of building open science practices and services within our university. This landscape is 
changing rapidly although it does seem that open science still appears to be mostly focused on open access publishing.

The researchers seem to be most interested in the issues that influence their daily work, i.e. data management plans and opening their publications. This is most likely due to the requirements of the main research funders and their policies that push them to take these steps. There is a need for the academic community to develop positive reward schemes for the inclusion of open science steps on the researcher's career ladder in order to make open science the norm, not the exception.

It seems that the culture of openness is still at the development stage at the UEF. The innovators, i.e. active research groups and researchers are already practicing and promoting openness, but the majority of the academic staff seems to be either unaware about open science or are unwilling to implement it due to the fact that incentives and other career advancement processes still support the traditional way of conducting research.

There is still much to do in terms of motivational work. It seems that the most important actors are the research groups and their leaders; these are the individuals that make the greatest impact. The individual academics seem also to be quite self-reliant and do not ask questions or attend lectures about the open science. Thus, it is very important that open science issues must be embedded in the students' core curriculum.

The library seems to be a suitable university unit to support and help researchers, perhaps due to its neutral service position within the university. The role of other university actors, for example the administration is still rather unfocused. In the future, improvements in this situation will require good planning before embarking on the appropriate activities.

\section{Availability of Data}

The full text of the questionnaire, the answers received from the respondents, and the saved data from the SPSS analysis are available in the LIBER Quarterly Dataverse (at the Harvard Dataverse): https://doi.org/10.7910/ DVN/SYYKD1. 


\section{Acknowledgement}

EOSC-Nordic has received funding from the European Union's Horizon 2020 research and innovation programme under grant agreement No 857652.

The authors are grateful to Dr. Ewen MacDonald for linguistic advice.

\section{References}

Archambault, É., Amyot, D., Deschamps, P., Nicol, A., Provencher, F., Rebout, L., \& Roberge, G. (2014). Proportion of Open access papers published in peer-reviewed journals at the European and world levels-1996-2013. RTD-B6-PP-2011-2: Study to develop a set of indicators to measure open access. Brussels: European Union. Retrieved September 3, 2020, from http://science-metrix.com/sites/default/files/science-metrix/ publications/d $1.8 \mathrm{sm}$ ec dg-rtd proportion oa 1996-2013 v11p.pdf.

Clifton, C. (2019). Data mining. Encyclopaedia Britannica. London: Encyclopaedia Britannica. Retrieved September 3, 2020, from https:/ / www.britannica.com/ technology/data-mining.

EOSC Summit. (2017). EOSC declaration. Brussels: European Open Science Cloud. Retrieved September 4, 2020, from https://ec.europa.eu/research/openscience/pdf/ eosc declaration.pdf.

European Commission. (2012). Commission recommendation of 17.7.2012 on access to and preservation of scientific information. Version C(2012) 4890 final 17.7.2012. Brussels: European Commission. Retrieved September 7, 2020, from http://ec.europa.eu/research/science-society/document library/pdf 06/ recommendation-access-and-preservation-scientific-information en.pdf.

European Commission. (2017). H2020 Programme: Guidelines to the rules on open access to scientific publications and open access to research data in Horizon 2020. Version 3.2. 21 March 2017. Brussels: European Commission. Retrieved September 3, 2020, from https://ec.europa.eu/research/participants/data/ref/h2020/grants_manual/hi/ oa pilot/h2020-hi-oa-pilot-guide en.pdf.

European Commission. (2018). Prompting an EOSC in practice. Brussels: European Commission. Retrieved September 4, 2020, from https://op.europa.eu/en/ publication-detail/-/publication/5253a1af-ee10-11e8-b690-01aa75ed71a1.

European Commission. (2019). EOSC main background documents: 20 August 2019. Brussels: European Commission. Retrieved September 4, 2020, from https:// ec.europa.eu/research/openscience/pdf/EOSC main background documents. pdf\#view=fit\&pagemode $=$ none. 
Forsström, P.-L., Lilja, E., \& Ala-Mantila, M. (2020). Atlas of open science and research in Finland 2019: Evaluation of openness in the activities of higher education institutions, research institutes, research-funding organisations, Finnish academic and cultural institutes abroad and learned societies and academies. Final report. Ministry of Education and Culture, Finland. Retrieved September 4, 2020, from http://urn.fi/ URN:ISBN:978-952-263-689-8

Ilva, J., Laitinen, M., \& Saarti, J. (2016). The costs of open and closed access: Using the Finnish research output as an example. LIBER Quarterly, 26(1), 13-27. https://doi. org/10.18352/lq.10137.

Kraker, P., Leony, D., Reinhardt, W., \& Beham, G. (2011). The case for open science in technology enhanced learning. International Journal of Technology Enhanced Learning, 3(6), 643-654. https://doi.org/10.1504/IJTEL.2011.045454.

Retrieved September 4, 2020, from https://pdfs.semanticscholar.org/773d/ c03085f19a5b861d0efcda5baabf25ed869a.pdf.

LERU. (2018). Open science and its role in universities: A roadmap for cultural change.

Retrieved September 4, 2020, from https:// www.leru.org/files/LERU-AP24-OpenScience-full-paper.pdf.

LIBER. (2018). Open science roadmap. Retrieved September 4, 2020, from https:// zenodo.org/record/1303002/files/LIBER OSR A5-ONLINE-HR.pdf.

Ogungbeni, J. I., Obiamalu, A. R., Ssemambo, S., \& Bazibu, C. M. (2016). The roles of academic libraries in propagating open science: A qualitative literature review. Information Development, 34(2), 113-121. https://doi.org/10.1177/0266666916678444.

Saarti, J., \& Tuominen, K. (2017). From paper-based towards post-digital scholarly publishing: An analysis of an ideological dilemma and its consequences. Information Research, 22(3), paper 769, n. p. Retrieved September 3, 2020, from http:// InformationR.net/ir/22-3/paper769.html.

Saarti, J. (2018). Collection policies for the post-digital era of academic publishing: Future scenarios for the academic libraries. In: Vattulainen, P., \& O'Connor, S. (Eds.), Repositories for print: Strategies for access, preservation and democracy -Current topics in library and information practice 9 (pp. 103-112). Berlin: De Gruyter Saur. https://doi. org/10.1515/9783110535372-010.

ScienceDirect. (2020). Data mining research. Amsterdam: Elsevier. Retrieved September 5, 2020, from https:/ / www.sciencedirect.com/topics/computer-science/ data-mining-research. 